\title{
Chronic kidney disease in Sri Lanka: a glimpse into lives of the affected
}

\author{
Sameera Senanayake \\ Australian Centre for Health Services Innovation, Queensland University of Technology, Brisbane, Australia \\ Correspondence: sam197902@gmail.com (iD https://orcid.org/0000-0002-5606-2046
}

DOI: https://doi.org/10.4038/jccpsl.v24i2.8158

Received on: 18 May 2018

Accepted on: 30 June 2018

"Chronic kidney disease of unknown aetiology is the beginning of a national catastrophe, perhaps unparalleled in recent history. The actions, non-actions and complacencies of the scientific community and medical profession will be judged by history".

- Jayasinghe S, CMJ 2014 -

Chronic kidney disease (CKD), taking the lives of thousands in Sri Lanka, is now considered a public health emergency. This article presents a critical analysis of the situation in Sri Lanka and a glimpse of CKD patients' suffering based on a community-based study conducted in the district of Anuradhapura. It provides an insight to the problem and paves way to solicit the support of individuals as well as the scientific communities and medical professionals to overcome this national catastrophe.

\section{Global and local burden of CKD}

Chronic kidney disease has been a well-known disease entity since ancient times. The medical definition of CKD requires many aspects to be fulfilled. There should be either a structural or functional abnormality of the kidney, which should have been present for more than three months, with implications to health (1).

The burden of CKD is increasing globally, while regional disparities exist. During the period of 19902010, the burden of CKD in the world has shown an annual growth rate of $8 \%$. Prevalence of those living with end stage renal disease is estimated to be high in developed countries (2); however, this is not a suitable indicator to measure the burden of CKD in developing countries as many do not survive up to end stage owing to the lack of dialysis facilities (3).
As with any other region of the world, the prevalence of CKD is on the rise in South East Asia. According to a recent study done in two major cities in India, Chennai and Delhi, the age standardized prevalence of CKD was found to be $8.7 \%$ (4). Over the last 25 years, the problem of CKD in Sri Lanka has drawn the attention of national and international medical communities, following an exponential increase noted in the number of CKD cases in the North Central Province (NCP) (5). More alarmingly, the fact that CKD in this province is not being due to any known cause is a great concern.

\section{Understanding CKDu through epidemiological principles}

Chronic kidney disease in which the cause cannot be attributed to any known aetiology is named CKD of uncertain aetiology (CKDu). Sri Lanka is not alone in this medical mystery. During the past 10-15 years, several regions in the world such as El Salvador, Nicaragua, Egypt and India have been affected (6-7). Different nomenclature has been used in the literature to label $\mathrm{CKDu}$, with the majority named after the region or country: Central American nephropathy, Salvadoran agricultural nephropathy, Mesoamerican epidemic nephropathy, chronic tubule-interstitial kidney disease of Central America and Udhanam endemic nephropathy in India (8-9). 
Though reported throughout the world, it is difficult to compare the CKDu prevalence between countries due to lack of a common case definition. In Sri Lanka, CKD has grown with the cause remaining unknown, taking thousands of lives in poor farming communities, and is now considered a humanitarian tragedy with grave implications on the society and economy. At the same time, while all the attention is drawn to 'unknown' CKD, the clinical evidence clearly indicates that the number of CKD patients with known causes such as hypertension, diabetes and glomerular nephritis has also increased over the years (10).

The epidemiological principles lead to clues on the origin of unknown diseases, based on exploration of time, person and place of the disease (11). The epidemiological profile of CKDu discovered thus far in Sri Lanka and other countries has not led to any definitive clues, precluding the epidemiologists from discovering the unknown even after more than two decades of existence of the disease.

It is evident that CKD of known aetiology is prevalent throughout the country and that CKD of unknown aetiology is highly prevalent in certain geographical areas of Sri Lanka. However, valid estimates of the prevalence of CKD/CKDu are not available for Sri Lanka. Therefore, understanding the burden of $\mathrm{CKD} / \mathrm{CKDu}$ in the country requires a robust community-based surveillance system which not only estimates the prevalence but also maps the distribution of cases according to geographical locations and person characteristics.

\section{Sources of data of CKD/CKDu in Sri Lanka}

The routine sources of morbidity and mortality data in Sri Lanka do not provide comprehensive information for estimating the CKD burden in Sri Lanka.

\section{- Indoor Morbidity and Mortality Return (IMMR)}

- IMMR is a collation of records on the causes for hospitalization and death of patients admitted to state hospitals in the country, which is published annually as the Annual Health Bulletin. It uses an internationally agreed upon system to record the cause but does not have a specific category on CKD/CKDu. It reports both acute as well as chronic renal failure together as 'renal failure'.

There are two registries in Sri Lanka that serve as databases for diagnosed CKD/CKDu patients under care.
- National Renal Registry - National Renal Registry is a web-based registry maintained by the Epidemiology Unit since 2013. Whenever a patient is diagnosed as having CKD or CKDu by a nephrologist or at a renal clinic designated as a sentinel site, information about the patient should be entered in the registry. The shortcoming in this registry is the incompleteness of data recording due to various logistical issues.

- Renal Registry Sri Lanka - Renal Registry is maintained by the Sri Lanka Society of Nephrologists, which is a collaborative effort of several stakeholders, including the Renal Disease Prevention and Research Unit and National Intensive Care Surveillance of Ministry of Health. This registry captures the information of CKD patients on dialysis from selected state institutions.

As CKD/CKDu patients may remain asymptomatic till late stages of the disease, data of the patients under care in clinics or hospitals cannot be expected to generate an accurate estimate of its prevalence.

\section{- Community-based screening programme -} Screening is a form of testing conducted among apparently well people to identify early asymptomatic diseases. Applying this epidemiological principle, the Ministry of Health regularly conducts communitybased screening programmes in areas designated as 'high risk' for CKD in the country. Currently, it is conducted in 12 districts. In Anuradhapura and Polonnaruwa districts, all divisional secretariat (DS) divisions are included in the programme, while in other districts it is only in a few selected DS divisions. This programme has been conducted since 2014 and during the year 2016, a total of 300,375 have been screened.

This method captures the data on only the screened positives, and does not differentiate between the CKD and CKDu cases. Further, these screening programmes have faced the challenge of not being able to get the participation of all 'at risk' individuals in a given geographical location. Though many measures have been taken, their participation has been less than $50 \%$.

- Special house to house survey in 2015 and 2016 in the designated 'high risk' areas - With the intention of fulfilling the gap in CKDu patient data, a special house to house survey is conducted by the 
Ministry of Health in all areas designated as 'high risk', to extract $\mathrm{CKD} / \mathrm{CKD}$ u status from medical records. A total of 24,806 patients have been identified from 11 high risk districts, of which 9,902 were from Anuradhapura district. However, information is only gathered from those already diagnosed as $\mathrm{CKD} / \mathrm{CKDu}$ while there is no information on those who are asymptomatic and undiagnosed, which has prevented calculating meaningful prevalence estimates through these special surveys.

- Research evidence - A few researchers have taken up community-based surveys in several geographical locations to assess the prevalence of CKDu/CKD. The main issue in comparing these estimates is the variation in case definitions used to identify CKDu.

In summary, none of the existing sources of data in Sri Lanka is able to provide a complete understanding of the problem of CKD in terms of its magnitude, distribution and trends, and the proportional contribution of CKDu to the total CKD burden. This means, though the country has been experiencing an epidemic of CKDu for over two decades which has attracted the attention of political leaders, media, policy makers and the public, the extent of the issue in hand is not known to date.

\section{Clues on the 'unknown' of CKDu}

Causality of a disease in epidemiology requires fulfilment of a set of criteria which require a plethora of evidence drawn from well-designed epidemiological studies repeated over time. The first step is to formulate hypotheses based on available information while the next step is to prove or disprove it using appropriate epidemiological study designs, preferably a prospective cohort study. However, determining the cause needs further epidemiological evidence on 'causality' (12).

In this backdrop, multiple hypotheses have been formulated on potential risk factors for CKDu in Sri Lanka and in other countries, though none have provided enough evidence to identify the exact cause.

- Cadmium (Cd) - Several studies, including the study conducted by the World Health Organization (WHO) in 2013 (13) evidenced that the possible cause of CKDu in NCP is due to high Cd levels found in soil, sediment from reservoirs, rice, fresh water fish and lotus roots. However, the findings were not consistent when repeated (14-15).
- Arsenic - High content of arsenic in drinking water due to the excessive use of fertilizers and pesticides, is another postulated risk factor that received considerable coverage in the media (16). This finding provoked a significant debate in the scientific community, which questioned the methodology used by the researchers.

- Glyphosate - Formation of glyphosate (a systemic organophosphate herbicide and heavy metal complexes in the presence of hard water has been hypothesized as the cause for CKDu in NCP. According to the authors, the most likely heavy metal causing kidney damage is arsenic (17). However, these findings have not been consistent across different studies to draw definitive conclusions.

- Fluoride - Presence of a high content of fluoride in drinking water and the use of substandard aluminium pots for cooking and storing water were two other theories that came up (18-19). However, this theory too failed to show evidence on fluoride as the cause.

- Dehydration - Excessive sweating and insufficient water and mineral replacement among farmers have been postulated as the cause for Mesoamerican nephropathy in Central America; and epidemiological studies are underway to test this hypothesis (20). The available evidence and circumstances do not completely rule out heat stress as the possible cause of CKDu in Sri Lanka.

As of today, most scientists in Sri Lanka accept the hypothesis that CKDu is likely to be multifactorial rather than due to a single cause.

Complexity of the epidemiological problem at hand and the lack of clearly designed research agenda have sadly kept us from discovering the 'unknown' in spite of 25 years of research. Non-uniform definitions to differentiate CKDu from CKD, studies not supported by appropriate study designs for generating valid data on causality, individual groups only interested in promoting their own theories in the absence of valid evidence and not critically evaluating the role of multiple factors acting together to produce the disease are some of the reasons for this state.

The current need is a consorted effort by a multidisciplinary group of researchers to conduct the most appropriate epidemiological study using a prospective cohort design, which takes into account all the potential 
risk factors that have been hypothesized in a single study. The Ministry of Health supported by the National Science Foundation and WHO has made progress recently in this regard, by arriving at a three-level case definition for CKDu through consensus of the members of Sri Lanka Society of Nephrologists, scientists, epidemiologists and researchers in October 2016. It has been published as a circular and disseminated in 2016 for the use by clinicians and researchers. Furthermore, Epidemiology Unit in collaboration with the National Science Foundation, WHO and Sri Lanka Society of Nephrologists, has implemented a large-scale community-based survey to generate valid estimates on the prevalence of CKDu, which will serve as the baseline for a prospective cohort study to discover the unknown in the coming years. Another effort to harmonize the CKD related research in the country is the joint effort by the Gardiner International Society of Nephrology Foundation, Presidential Task Force for Prevention of CKD, University Grants Commission and University of Colombo to establish a consortium of national/ international researchers to conduct long-term interdisciplinary research to better understand the aetiology of CKDu.

\section{Action by different sectors on CKD/CKDu in Sri Lanka}

Absence of accurate data does not seem to have hindered the country in responding to the problem of $\mathrm{CKD} / \mathrm{CKDu}$. The response from different sectors has been prompt and considerable.

\section{Government response}

Establishment of the Presidential Task Force for the Prevention of CKDu in the year 2014 highlights the fact that the government realizes the urgency as well as the importance of centralized coordination. Aim of this task force is to ensure the eradication of CKDu through a coordinated process. Further, the National Kidney Fund has been established under the purview of the task force and the funds are being utilized to construct palliative care units and to award education scholarships for G.C.E Advanced Level students of families whose parents have died of the disease.

The political establishment acted promptly in the year 2015 in imposing a ban on pesticides especially herbicide glyphosate, based on evidence of the association of arsenic and CKDu in a single initial study.
Two popular sportsmen have been appointed as brand ambassadors by His Excellency the President to support the care services. The Government response thus far has been mainly through the following ministries.

- Ministry of Health programme - The Ministry of Health established a CKD focal point in 2013 under the deputy director general of public health services to coordinate the CKD related activities within the ministry as well as between different ministries.

The main contribution has been on community screening of at-risk population in endemic areas to detect $\mathrm{CKD} / \mathrm{CKDu}$ cases early and to direct them for early care implemented through provincial health services and to expand treatment facilities in affected areas.

- Ministry of Agriculture - The Ministry of Agriculture programme primarily targets reduction of irrational and overuse of agrochemicals.

- Ministry of Water Supply and Drainage - The Ministry of Water Supply and Drainage principally expanded the supply of safe water. The long-term objective is provision of pipe-borne water while the short-term provisions are distribution of safe drinking water using bowsers and tanks filled by the National Water Board and groundwater purification by reverse osmosis plants.

- Ministry of Social Services Programme - The Ministry of Social Services provides social services to the affected people by paying a monthly assistance of Rs. 3000 each for patients who require dialysis.

\section{International response}

As the problem escalated, international communities and many governments reached out to Sri Lanka for assistance. Among the latest support is the fully equipped hospital dedicated to renal diseases in Polonnaruwa being built by the Chinese Government. Further, the Gardiner Foundation in Sri Lanka has tied up with the International Society of Nephrology to commence, not just a Sri Lankan, but a global mission to initiate and ensure the sustainability of multi-faceted activities for prevention and treatment of CKD.

As highlighted, responses from different sectors on the problem of CKDu have been commendable, 
though the coordination of actions to ensure that efforts complement each other has not been optimal. Though many of these responses are towards eliminating the suspected risk factors of $\mathrm{CKDu}$, none of these responses are linked with an assessment to evaluate its effectiveness in doing so, losing a golden opportunity to generate the essential evidence required to prove 'causality'.

\section{Glimpse of the suffering of $\mathrm{CKD} / \mathrm{CKDu}$ patients}

Evidence on the suffering of CKD patients, comes largely from a research conducted during October 2015-March 2016 among 1118 CKD patients living in Anuradhapura district in the year 2016. Its aim was to describe the burden of symptoms, psychological problems, disease related quality of life and household cost incurred by diagnosed CKD patients.

Patients confirmed as having $\mathrm{CKD} / \mathrm{CKDu}$ and registered in the government renal clinics in NCP were the eligible study units. The study was conducted as a household survey in all 19 medical officer of health $(\mathrm{MOH})$ divisions in the district of Anuradhapura. The number of patients included from each $\mathrm{MOH}$ was proportionate to the number of registered patients in the $\mathrm{MOH}$ area. The required number of patients to be included from each $\mathrm{MOH}$ area was selected using a simple random sampling technique using the updated list of registered patients as the sampling frame.

Approximately half of the study population (51.2\%) was in the 41-60 year age category, with the majority being males (62.7\%). More than half the population (56.3\%) were in CKD stage IV, indicating late stages of the disease. Based on the recorded information on diagnosis, the proportion of CKDu patients among all was $43.7 \%$.

\section{Physical suffering}

Over the last few decades, the medical efforts on symptom management and end-of-life strategies were mainly focusing on cancer patients. However, symptom distress of CKD from the perspective of the patients could be much worse (21-22).

In an attempt to present a holistic picture of the suffering, the study assessed prevalence, severity and burden of symptoms experienced by the CKD patients. As part of the study, a culturally sensitive tool was developed, Chronic Kidney Disease Symptom Index Sri Lanka and validated it among a sample of 250 randomly selected CKD patients attending the five renal clinics in Polonnaruwa district (23). Results showed that this tool is a valid and reliable too to assess the symptom burden in different CKD populations.

The burden of symptoms was assessed using this validated tool on 1118 study units. The results indicated that among the symptoms assessed, bone/joint pain, feeling irritable, muscle cramps and lack of energy were the most prevalent. Of the 25 symptoms inquired into, 22 were more prevalent among the older age group and 21 experienced by more females indicating that the suffering was more among the old and females. Analysis of the symptom burden by the stage of CKD showed that the prevalence was similar and high, irrespective of the stage. Perceived severity of symptoms showed that most severe symptom among both males and females was loss/ decreased libido (24).

An assessment of the symptom burden among CKD patients in Sri Lanka has not been carried out prior to this study, indicating the low priority given to patients' perspectives of the suffering.

Chronic Kidney Disease Symptom Index - Sri Lanka developed and validated by Senanayake et al. allowed the quantification of symptom burden using a composite score which accounted for both prevalence and perceived severity of symptoms. This tool could be used in the clinical practice as well as for research purposes in the future.

The study also showed that $98.0 \%$ attended a clinic in a government health institution. Considering that a great majority had accessed care but were still experiencing a high prevalence of symptoms, may be an indication of inadequate attention by the service providers to relieve patients of their symptom burden. In most of the developed countries, organized systems provide pharmacological and non-pharmacological remedies specifically designed to relieve CKD patients of their symptoms.

\section{Psychological suffering}

A person with CKD could experience several stressors in life due to the effect of illness, changes in family dynamics, dietary restrictions and functional limitations. Depression is commonly associated with chronic diseases. Evidence suggests that CKD patients 
with depressive symptoms have adverse health outcomes (25-27).

The study attempted to assess the psychological suffering of CKD patients in Anuradhapura district, by screening all the study units for depression using the CES-D questionnaire and for psychological distress using GHQ-12. Both tools have been previously validated for adults in Sri Lankan setting (28-29).

The findings showed alarming results. A great majority (65.2\%; 95\% CI=62.4, 68.0) was screened positive for depression while three $(75.0 \%$; $95 \%$ $\mathrm{CI}=72.5,77.6)$ showed evidence of psychological distress (30).

The prevalence of depression in developed countries among CKD patients is much lower than that of the estimates in Sri Lanka. Availability of standard psychological support as an integral part of the care package provided to these patients is said to be the reason (31). Such psychological support entails regular screening for depression and diagnosis and treatment of the affected. The unavailability of such organized screening services and follow up diagnostic and management can be the reason for the high prevalence in Sri Lanka.

The study results indicate the need for organized screening system for psychological conditions with supportive diagnostic and management services at least in CKD affected areas. Psychotherapy is an effective treatment for early stages of clinical depression, thus all who are depressed do not need drugs. Therefore, the inadequate psychological care provided to these patients cannot be dismissed with the excuse of not having adequate specialist psychiatrists in Sri Lanka.

Another gap identified was patients not being aware of the available mental health services. This was evident by only $14.9 \%$ of the depressed patients knowing the mental health services provided in government hospitals. This highlights the fact that the health system has failed in educating the patients regarding the available health resources in this part of the country.

\section{Quality of life}

Importance of QOL measurements in relation to CKD patients has come to the limelight in the recent past (32-33). Different instruments have been used worldwide to measure QOL among CKD patients, of which Kidney Disease QOL Short Form has been a widely used tool comprising two components; kidney disease specific and SF 36. This tool was culturally adapted and validated as part of the study, which confirmed it as a reliable and valid instrument for assessing the QOL among CKD patients (34).

Results revealed that higher educational status and being employed were independently associated with better QOL, while being depressed, psychologically distressed, having high symptom burden and being on dialysis were independently associated with poor QOL. This highlights that improvement of quality of life requires physical symptom alleviating measures as well as measures to improve the mental health status.

\section{Social suffering}

CKD directly impacts on patients' everyday life including their livelihood, domestic tasks and participation in social activities. Such an illness brings about drastic changes within the family in relation to resource allocation, consumption patterns and maintaining social relationships, and subsequently within the entire community.

\section{Suffering of the patients}

The study was enriched by in-depth interviews conducted to obtain an insight into the impact of the disease on daily life. Inquiries revealed that following the diagnosis of CKD, most patients struggle to continue with their same lifestyle. They are advised on changing over to healthy lifestyles with no practical tips on how to adopt them within their life situations. Wanting a mean of living, they are compelled to continue with the same job often with no skills or means to engage in another job. The common story is a gradual deterioration of productivity until being bedridden.

The social network of patients is active during the initial stages of the diagnosis when people visit them with the good intention of providing them and the family with both emotional and material support. However, these social connections gradually diminish as the disease progresses and reappear when the illness becomes critical.

CKD is strongly associated with stigma to the extent that finding marriage partners for children of the patients has been problematic in some high prevalent 
areas. Thus, patients tend to hide the disease from the society and at times neglect the required medical attention.

\section{Suffering of the family}

Lives of the patient's family are affected in several ways. The family is affected first, by low productivity of the sick member; second, by allocating resources both human and material to manage ill-health; and third, by the emotional and social cost of illness on the family. Family members need to take on the patient's workload, along with the load of caring for the patient. In some families, members had to give up their livelihood in order to provide necessary care for the patient.

\section{Economic suffering}

CKD causes a great economic impact on the patients. Disability, work loss and out-of-pocket expenditure borne by households on these patients have adverse effects not only on the socioeconomic status but also on the psychological status of patients and their families.

In the study, household cost of accessing health services was assessed among 1118 CKD patients in Anuradhapura District (35). Quite understandably, their income had reduced significantly since the illness. This was more intensely seen among farmers (80\%). Farming needs strenuous physical labour and with the symptomatology present even in the early stages of the disease, it could make working extremely challenging.

\section{Household cost for hospital admissions}

The study gathered data on household costs from the CKD patients who had been admitted to hospital during the last six months. There were 59 such patients. Total average household cost for a single hospital admission was Rs. 3700. The proportion of patients who had to pay for drugs and investigations during the hospital stay and the amount they had to pay were high. Presumably the shortage of drugs and lack of facilities to perform investigations had resulted in this high out-of-pocket expenditure.

\section{Household cost for a clinic visit}

Nearly quarter of the study population had travelled 26-50 km to attend a government clinic. Some had travelled to clinics located in adjacent districts. This was either due to unavailability of a closer renal clinic or bypassing the nearby health institutions in search of better care. These are major concerns which warrant urgent attention of the policy makers.

The median time spent to obtain services in a government clinic was nearly 8 hours, of which the median time taken to consult a doctor was three hours. The highest waiting time was 4 hours reported from Anuradhapura Teaching Hospital. Further, the household cost accounting for the total direct cost and lost income of the patient for a single clinic visit was Rs.500. The highest total cost was borne by those visiting Anuradhapura Teaching Hospital (Rs.1102.8; $\mathrm{IQR}=400-1267.5$ ).

\section{Household cost for dialysis patients}

There were 39 patients on dialysis, of whom more than half were living 25-50 km away from the dialysis centre, highlighting their limited accessibility to care. The median time spent on a dialysis episode was more than 10 hours. This is considered significantly long as these patients undergo dialysis either two or three times per week. A dialysis patient spends nearly half of the total cost incurred on transport. Further, he spends approximately three hours on travelling, thus traveling imposing a significant cost and time burden on the CKD patients. The total monthly household cost for a dialysis patient was Rs.6807.

Dialysis patients suffer the highest symptom burden, depression and psychological distress and the lowest quality of life. On top, the substantial financial burden faced by the dialysis patients, further aggravates their living standards.

\section{Perception regarding kidney transplant}

The option of kidney transplant as a treatment modality had been suggested to $12.2 \%$ of the participants. Of them, only $29.4 \%$ have considered it as a feasible option (36). Qualitative inquiries revealed that having to find a suitable live donor and the economic and social difficulties associated with this process was the main underlying reason. Sibling/child donors as being the most feasible option for patients of CKD with known causes, is in fact not suitable for CKDu patients with their constant fear of the family members being diagnosed with the condition sooner or later. These findings are further supported by a study done among patients in stages 4 and 5 attending a low clearance clinic in Polonnaruwa District General Hospital. Only $8.4 \%$ of the patients had taken the final 
decision to undergo transplant. A great majority (83.3\%) had decided not to undergo it owing to limited finances (36). Though kidney transplant services were successfully initiated in Sri Lanka over 30 years back, an organized mechanism of cadaveric transplantation has not been established. With the responsibility of identifying a suitable donor being made the responsibility of the patient and family who are already hampered with ill health, mental suffering and economically hardships, kidney transplant has become an unpopular choice.

\section{Minimizing the catastrophe}

A few key areas need attention of the scientific communities and medical professionals towards overcoming this national catastrophe.

- Researchers of different disciplines need to get together and design and contribute to a common research agenda that generate evidence on the causality of CKDu

- The interventional responses should be better coordinated to ensure that efforts complement each other and are linked with assessments that evaluate the effectiveness

- There are several measures that can be taken by health and social service sectors to overcome or minimize the suffering of the patients.

a. Health sector should focus its attention on symptom alleviating therapies to improve the psychological wellbeing of CKD patients

b. Measures to reduce household costs when accessing healthcare services need to be explored and implemented

c. It is imperative that kidney transplant services are re-organized in a manner that will be more acceptable to the patients and families without further burdening them

d. Social service sector should focus their attention on designing and implementing alternative employment or income generation methods to the diagnosed patients and on measures to lower the household cost related to CKD.

Acknowledgement: Dr Nalika Gunawardena for the technical guidance.

\section{Competing interests: None}

\section{References}

1. Bailie GR, Uhlig K, Levey AS. Clinical practice guidelines in nephrology: evaluation, classification and stratification of chronic kidney disease. Pharmacotherapy: the Journal of Human Pharmacology and Drug Therapy 2005; 25(4): 491-502.

2. Alebiosu CO, Ayodele OE. The global burden of chronic kidney disease and the way forward. Ethnicity \& Disease 2005; 15(3): 418-423.

3. Barsoum RS. Chronic kidney disease in the developing world. New England Journal of Medicine 2006; 354(10): 997-999.

4. Anand S, Shivashankar R, Ali MK, Kondal D, Binukumar B, Montez-Rath ME, et al. Prevalence of chronic kidney disease in two major Indian cities and projections for associated cardiovascular disease. Kidney International 2015; 88(1): 178-185.

5. Jayasekara JM, Dissanayake DM, Adhikari SB, Bandara P. Geographical distribution of chronic kidney disease of unknown origin in North Central Region of Sri Lanka. Ceylon Medical Journal 2013; 58(1): 6-10.

6. Sanoff SL, Callejas L, Alonso CD, Hu Y, Colindres RE, Chin H, Morgan DR, Hogan SL. Positive association of renal insufficiency with agriculture employment and unregulated alcohol consumption in Nicaragua. Renal Failure 2010; 32(7): 766-767.

7. Trabanino RG, Aguilar R, Silva CR, Mercado MO, Merino RL. End-stage renal disease among patients in a referral hospital in El Salvador. Revista Panamericana de Salud Pública 2002; 12(3): 202-206.

8. Correa-Rotter R, Wesseling C, Johnson RJ. CKD of unknown origin in Central America: the case for a mesoamerican nephropathy. American Journal of Kidney Diseases 2014; 63(3): 506-520.

9. Almaguer M, Herrera R, Orantes CM. Chronic kidney disease of unknown etiology in agricultural communities. MEDICC Review 2014; 16(2): 9-15.

10. Gooneratne I, Ranaweera A, Liyanarachchi N, Gunawardane N, Lanerolle R. Epidemiology of chronic kidney disease in a Sri Lankan population. International Journal of Diabetes in Developing Countries 2008; 28(2): 60.

11. Hennekens CH, Buring JE, Mayrent SL. Epidemiology in Medicine (1 $1^{\text {st }}$ edition). Boston: Little, Brown, 1987.

12. Hill AB. The environment and disease: association or causation? Journal of the Royal Society of Medicine 2015; 108(1): 32-37. 
13. Jayatilake N, Mendis S, Maheepala P, Mehta FR. Chronic kidney disease of uncertain aetiology: prevalence and causative factors in a developing country. BMC Nephrology 2013; 14(1): 180.

14. Chandrajith R, Nanayakkara S, Itai K, Aturaliya T, Dissanayake C, Abeysekera T, et al. Chronic kidney diseases of uncertain etiology (CKDue) in Sri Lanka: geographic distribution and environmental implications. Environmental Geochemistry and Health 2011; 33(3): 267-278.

15. Bandara J, Senevirathna D, Dasanayake D, Herath V, Bandara J, Abeysekara T, et al. Chronic renal failure among farm families in cascade irrigation systems in Sri Lanka associated with elevated dietary cadmium levels in rice and freshwater fish (Tilapia). Environmental Geochemistry and Health 2008; 30(5): 465-478.

16. Jayasumana C, Paranagama P, Agampodi S, Wijewardane C, Gunatilake S, Siribaddana S. Drinking well water and occupational exposure to herbicides is associated with chronic kidney disease in PadaviSripura, Sri Lanka. Environmental Health 2015; 14(1): 6.

17. Jayasumana C, Gunatilake S, Senanayake P. Glyphosate, hard water and nephrotoxic metals: are they the culprits behind the epidemic of chronic kidney disease of unknown etiology in Sri Lanka? International Journal of Environmental Research and Public Health 2014; 11(2): 2125-2147.

18. Johnson S, Misra SS, Sahu R, Saxena P. Environmental contamination and its association with chronic kidney disease of unknown etiology in North Central Region of Sri Lanka. Centre for Science and Environment, New Delhi, 2012.

19. Ileperuma O, Dharmagunawardhane H, Herath K. Dissolution of aluminium from sub-standard utensils under high fluoride stress: a possible risk factor for chronic renal failure in the North-Central Province. Journal of the National Science Foundation of Sri Lanka 2009; 37(3): 219-222.

20. García-Trabanino R, Jarquín E, Wesseling C, Johnson RJ, González-Quiroz M, Weiss I, et al. Heat stress, dehydration, and kidney function in sugarcane cutters in El Salvador - a cross-shift study of workers at risk of Mesoamerican nephropathy. Environmental Research 2015; 142: 746-755.

21. Tranmer JE, Heyland D, Dudgeon D, Groll D, SquiresGraham M, Coulson K. Measuring the symptom experience of seriously ill cancer and noncancer hospitalized patients near the end of life with the memorial symptom assessment scale. Journal of Pain and Symptom Management 2003; 25(5): 420-429.
22. Anderson H, Ward C, Eardley A, Gomm S, Connolly $\mathrm{M}$, Coppinger $\mathrm{T}$, et al. The concerns of patients under palliative care and a heart failure clinic are not being met. Palliative Medicine 2001; 15(4): 279-286.

23. Senanayake S, Gunawardena N, Palihawadana P. Development of the chronic kidney disease symptom index - Sri Lanka; a symptom assessment instrument for chronic kidney disease patients. Journal of the Postgraduate Institute of Medicine 2017; 4(1): E38 $1-12$.

24. Senanayake S, Gunawardena N, Palihawadana P, Bandara P, Haniffa R, Karunarathna R, et al. Symptom burden in chronic kidney disease; a population based cross sectional study. BMC Nephrology 2017; 18(1): 228.

25. Chiang H-H, Guo H-R, Livneh H, Lu M-C, Yen M-L, Tsai T-Y. Increased risk of progression to dialysis or death in CKD patients with depressive symptoms: A prospective 3-year follow-up cohort study. Journal of Psychosomatic Research 2015; 79(3): 228-232.

26. Hedayati SS, Grambow SC, Szczech LA, Stechuchak KM, Allen AS, Bosworth HB. Physician-diagnosed depression as a correlate of hospitalizations in patients receiving long-term hemodialysis. American Journal of Kidney Diseases 2005; 46(4): 642-649.

27. Tavallaii SA, Ebrahimnia M, Shamspour N, Assari S. Effect of depression on health care utilization in patients with end-stage renal disease treated with hemodialysis. European Journal of Internal Medicine 2009; 20(4): 411-414.

28. Abeysena C, Jayawardana P, Peiris U. Factor structure and reliability of the 12-item Sinhala version of general health questionnaire. International Journal of Collaborative Research on Internal Medicine \& Public Health 2012; 4(8): 1606-1613.

29. Ferdinando KDAR. Prevalence and correlates of depression in adults of 25-45 years in Kalutara DDHS area. MD Thesis (Community Medicine). Colombo: Postgraduate Institute of Medicine, 2006.

30. Senanayake SJ. Health status, quality of life and out of pocket expenditure of chronic kidney disease patients in Anuradhapura District. MD Thesis (Community Medicine). Colombo: Postgraduate Institute of Medicine, 2016.

31. Tsang A, Von Korff M, Lee S, Alonso J, Karam E, Angermeyer MC, et al. Common chronic pain conditions in developed and developing countries: gender and age differences and comorbidity with depression-anxiety disorders. The Journal of Pain 2009; 9(10): 883-891. 
32. Mujais SK, Story K, Brouillette J, Takano T, Soroka S, Franek C, et al. Health-related quality of life in CKD patients: correlates and evolution over time. Clinical Journal of the American Society of Nephrology 2009; 4(8): 1293-1301.

33. Tsai Y-C, Hung C-C, Hwang S-J, Wang S-L, Hsiao S$\mathrm{M}$, Lin M-Y, et al. Quality of life predicts risks of endstage renal disease and mortality in patients with chronic kidney disease. Nephrology Dialysis Transplantation 2009; 25(5): 1621-1626.

34. Senanayake S, Gunawardena N, Palihawadana P, Kularatna S, Peiris TSG. Validity and reliability of the Sri Lankan version of the kidney disease quality of life questionnaire (KDQOL-SFTM). Health and Quality of Life Outcomes 2017; 15(1): 119.

35. Senanayake SJ, Gunawardena NS, Palihawadana P, Bandara S, Bandara P, Ranasinghe AU, Karunarathna RH, Kumara GP. Out-of-pocket expenditure in accessing healthcare services among chronic kidney disease patients in Anuradhapura District. Ceylon Medical Journal 2017; 62(2): 100-103.

36. Sandaruwanie PKS. Factors associated with the decision on kidney transplantation among advanced CKD patients attending District General Hospital Polonnaruwa. MSc Dissertation (Community Medicine). Colombo: Postgraduate Institute of Medicine, 2017. 